\title{
Claudin-16 and claudin-19 interact and form a cation-selective tight junction complex
}

\author{
Jianghui Hou, ${ }^{1}$ Aparna Renigunta, ${ }^{2}$ Martin Konrad,, ${ }^{3}$ Antonio S. Gomes, ${ }^{1}$ Eveline E. Schneeberger, ${ }^{4}$ \\ David L. Paul, ${ }^{5}$ Siegfried Waldegger, ${ }^{2}$ and Daniel A. Goodenough 1 \\ 1Department of Cell Biology, Harvard Medical School, Boston, Massachusetts, USA. 2University Children's Hospital, Philipps-University Marburg, \\ Marburg, Germany. ${ }^{3}$ Department of Pediatrics, University of Münster, Münster, Germany. ${ }^{4}$ Molecular Pathology Unit, \\ Massachusetts General Hospital, Charlestown, Massachusetts, USA. ${ }^{5}$ Department of Neurobiology, Harvard Medical School, Boston, Massachusetts, USA.
}

\begin{abstract}
Tight junctions (TJs) play a key role in mediating paracellular ion reabsorption in the kidney. Familial hypomagnesemia with hypercalciuria and nephrocalcinosis (FHHNC) is an inherited disorder caused by mutations in the genes encoding the TJ proteins claudin-16 (CLDN16) and CLDN19; however, the mechanisms underlying the roles of these claudins in mediating paracellular ion reabsorption in the kidney are not understood. Here we showed that in pig kidney epithelial cells, CLDN19 functioned as a $\mathrm{Cl}^{-}$blocker, whereas CLDN16 functioned as a $\mathrm{Na}^{+}$channel. Mutant forms of CLDN19 that are associated with FHHNC were unable to block $\mathrm{Cl}^{-}$permeation. Coexpression of CLDN16 and CLDN19 generated cation selectivity of the TJ in a synergistic manner, and CLDN16 and CLDN19 were observed to interact using several criteria. In addition, disruption of this interaction by introduction of FHHNC-causing mutant forms of either CLDN16 or CLDN19 abolished their synergistic effect. Our data show that CLDN16 interacts with CLDN19 and that their association confers a $\mathrm{TJ}$ with cation selectivity, suggesting a mechanism for the role of mutant forms of CLDN16 and CLDN19 in the development of FHHNC.
\end{abstract}

\section{Introduction}

The human renal disorder familial hypomagnesemia with hypercalciuria and nephrocalcinosis (FHHNC; OMIM 248250) is characterized by progressive renal $\mathrm{Mg}^{2+}$ and $\mathrm{Ca}^{2+}$ wasting leading to impaired renal function and chronic renal failure. FHHNC has been genetically linked to mutations in the gene of claudin-16 (CLDN16, also known as paracellin-1) (1) and more recently to CLDN19 (2). The claudins comprise a 22-gene family that encodes essential structural proteins of the tight junction (TJ), which are the principal regulators of paracellular permeability. In vitro studies have shown that ion selectivity of the paracellular conductance (reviewed in ref. 3 ) is a complex function of claudin subtype and cellular context $(4,5)$.

In vitro analyses using cultured cell models show that CLDN16 plays a key role in maintaining the cation selectivity of the TJ and forms a nonselective paracellular cation channel (4). This hypothesis of a nonselective paracellular cation channel is supported by (a) a clinical study to correlate the cellular functions of CLDN16 mutations identified in FHHNC to the phenotypes of FHHNC patients, with a special focus on the progression of renal failure (6), and by (b) our mouse models using transgenic RNAi depletion of CLDN16 (7). Without CLDN16 expression in the kidney, TJs in the thick ascending limb (TAL) of the nephron lose cation selectivity, leading to the dissipation of the lumen-positive potential with a concomitant loss of the driving force for $\mathrm{Mg}^{2+}$ reabsorption (7). While targeted deletion of CLDN19 in mice initially focused on its

Nonstandard abbreviations used: CLDN, claudin; Cub, C-terminal half of ubiquitin; DPBS, Dulbecco PBS; FHHNC, familial hypomagnesemia with hypercalciuria and nephrocalcinosis; MDCK, Madin-Darby canine kidney (cell); Nub, N-terminal half of ubiquitin; $\mathrm{P}_{\mathrm{Cl}}$, absolute $\mathrm{Cl}^{-}$permeability; $\mathrm{P}_{\mathrm{Mg}}$, absolute $\mathrm{Mg}^{2+}$ permeability; $\mathrm{P}_{\mathrm{Na}}$, absolute $\mathrm{Na}^{+}$permeability; TAL, thick ascending limb; TER, transepithelial resistance; $\mathrm{TF}$, transcriptional factor; TJ, tight junction; $\mathrm{Y} 2 \mathrm{H}$, yeast 2-hybrid.

Conflict of interest: The authors have declared that no conflict of interest exists. Citation for this article: J. Clin. Invest. 118:619-628 (2008). doi:10.1172/JCI33970. role in peripheral myelin (8), promoter analysis (9) and subsequent studies $(10,11)$ have emphasized the presence of CLDN19 in TAL of the nephron (colocalizing with CLDN16). The renal function of CLDN19 and its role in the pathogenesis of FHHNC are not known. In the present study, we provide evidence that CLDN19 is involved in the control of ion selectivity of the TJ and identify a locus of amino acids critical for its function.

Claudins interact with each other both intracellularly and intercellularly: they copolymerize linearly within the plasma membrane of the cell, together with the integral protein occludin, to form the classical intramembrane fibrils or strands visible in freeze-fracture replicas. These intramembrane interactions (side-to-side) can involve 1 claudin protein (homomeric or homopolymeric) or different claudins (heteromeric or heteropolymeric). We will refer to intramembranous claudin-claudin interactions in this report as being homo- or heteromeric, mindful that the subunit structure of the TJ fibril is unknown (12). In the formation of the intercellular junction, claudins may interact head-to-head with claudins in an adjacent cell, generating both homotypic and heterotypic claudinclaudin interactions (13). Peripheral membrane proteins, such as ZO-1 (14) and ZO-2 (15), play key roles in recruiting claudins to $\mathrm{TJ}$ strands in polarized epithelial cells (16), but are not required in fibroblasts (13). Here we examined the physical interactions between CLDN16 and CLDN19 and provide evidence that their interaction generates a cation-selective TJ, underlying mechanisms of FHHNC pathogenesis.

\section{Results}

Expression of CLDN16, CLDN19, and their mutants. The expression and characterization of CLDN16 and its mutants has been described previously (4). To determine the function of CLDN19, we stably expressed CLDN19 in well-established epithelial cell models (e.g., LLC-PK1 and Madin-Darby canine kidney [MDCK] cells). Neither of these cell lines expressed endogenous CLDN19 


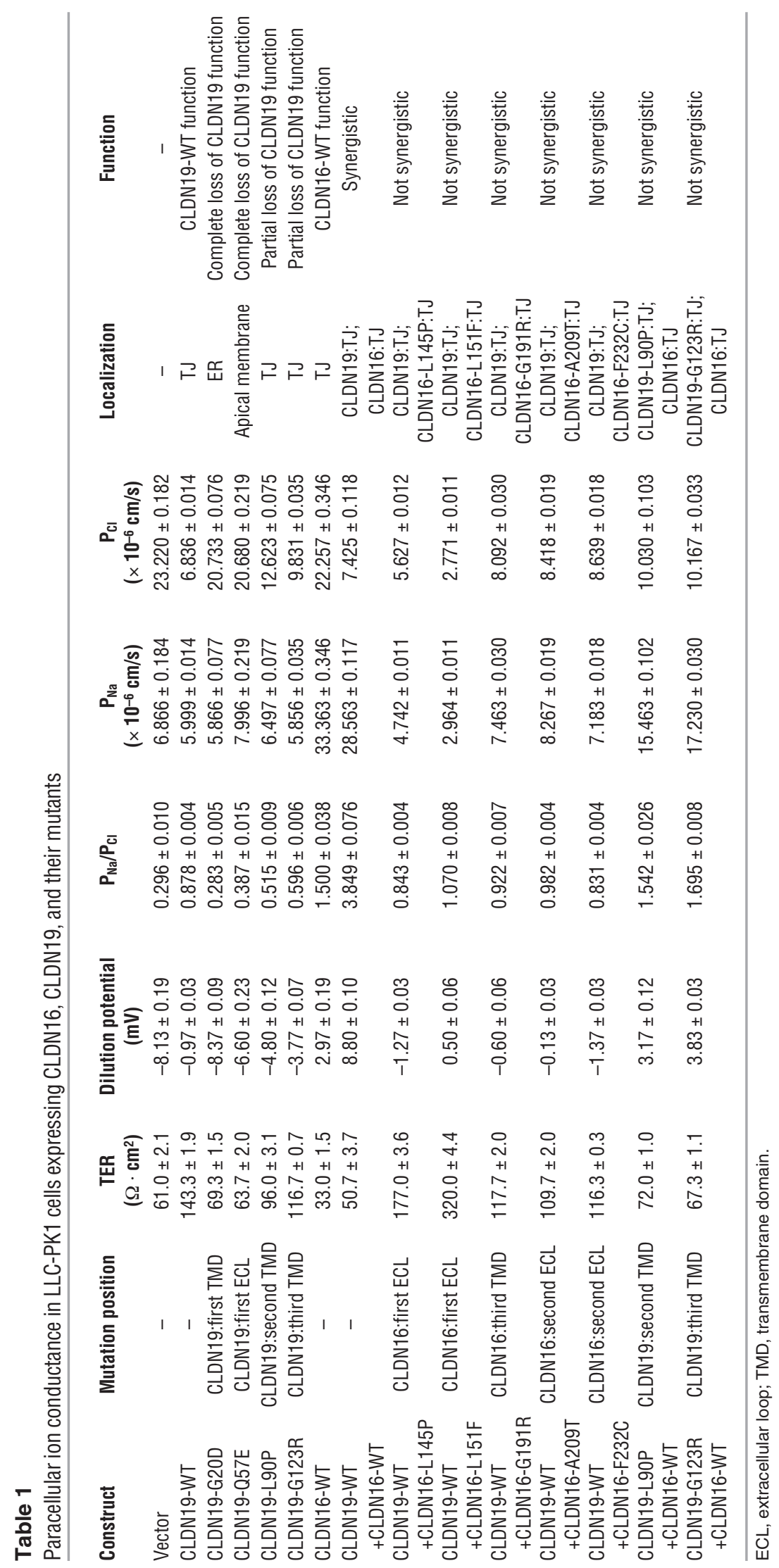

(control cells were infected with empty vector). Immunoblotting and immunostaining with CLDN19 antibody showed its electrophoretic mobility (as a $25-\mathrm{kDa}$ band) and its localization in the TJ, respectively (Supplemental Figure 1, A and C, showing LLCPK1 cells as a representative; supplemental material available online with this article; doi:10.1172/JCI33970DS1). As we aimed to have cells expressing CLDN19 over a prolonged period so that they could become fully polarized and form TJs, we used a previously described retroviral expression system to drive exogenous CLDN19 expression (4). LLC-PK1 and MDCK cells were infected individually with WT CLDN16 or CLDN19 or their mutants (vide infra). To normalize the expression among various mutants and with the WT, we infected cells with a fixed titer of virus at $1 \times 10^{6} \mathrm{CFU} / \mathrm{ml}$ and quantified the transcription of transgene by RT-PCR. On day 9 following the development of polarized monolayers, the Transwell filters were subjected to electrophysiological measurements and subsequently immunostained to visualize claudin expression. Cells coexpressing CLDN16 and CLDN19 were generated with sequential viral infections. The expression of exogenous CLDN16 (4) or CLDN19 had no effects on the expression levels of other endogenous claudins (CLDN1-CLDN4 and CLDN7 in MDCK cells; CLDN1, CLDN3, CLDN4, and CLDN7 in LLC-PK1 cells, which do not express CLDN2).

CLDN19 reduces $\mathrm{Cl}^{-}$permeation. In LLC-PK1 cells, we found that CLDN19 profoundly decreased absolute $\mathrm{Cl}^{-}$permeability $\left(\mathrm{P}_{\mathrm{Cl}}\right)$ without significant effects on absolute $\mathrm{Na}^{+}$ permeability $\left(\mathrm{P}_{\mathrm{Na}}\right.$; Table 1$)$. An apical-tobasal chemical gradient generated a -8.13 $\pm 0.19 \mathrm{mV}$ diffusion potential across the LLC-PK1 control monolayer (with the apical side as zero reference), indicating that the junctional pores of LLC-PK1 cells were more permeable to anions than cations. CLDN19 significantly increased the diffusion potential to $-0.97 \pm 0.03 \mathrm{mV}(n=3$, $P<0.01$; Figure 1A). The Goldman-Hodgkin-Katz equation calculated the ion permeability ratio $\left(\mathrm{P}_{\mathrm{Na}} / \mathrm{P}_{\mathrm{Cl}}\right)$ at $0.296 \pm 0.010 \mathrm{in}$ control cells compared with $0.878 \pm 0.004$ in cells expressing CLDN19 $(P<0.01$; Figure 1B). Transepithelial resistance (TER) was significantly increased by CLDN19 in LLC-PK1 cells, owing to its suppression of $\mathrm{Cl}^{-}$flux $\left(\mathrm{CLDN} 19,6.836 \pm 0.014 \times 10^{-6}\right.$ $\mathrm{cm} / \mathrm{s}$; control, $23.220 \pm 0.182 \times 10^{-6} \mathrm{~cm} / \mathrm{s}$; $P<0.01)$. Inhibiting the basolateral $\mathrm{Na}^{+} /$ $\mathrm{K}^{+}$-ATPase (1 mM ouabain) had no effects 
A

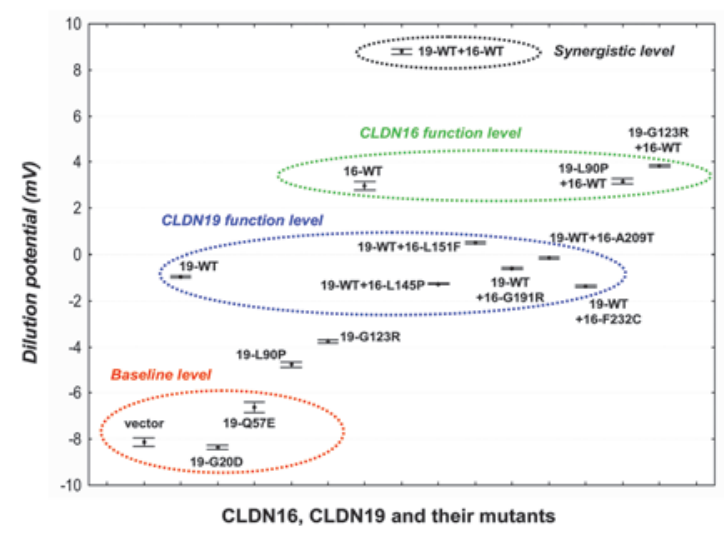

B

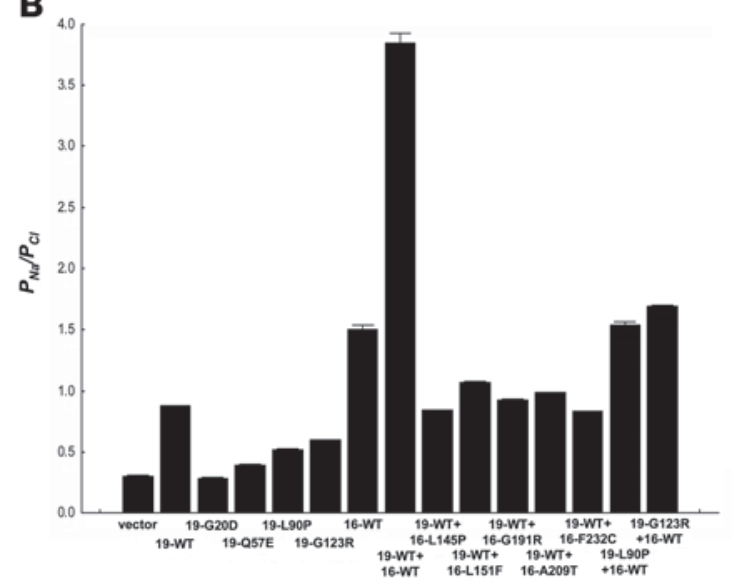

Figure 1

Effects of CLDN16, CLDN19, and their mutants in LLC-PK1 cells on paracellular ion conductance. Dilution potential values $(\mathbf{A})$ and $\mathrm{P}_{\mathrm{Na}} / \mathrm{P}_{\mathrm{Cl}}(\mathbf{B})$ across LLC-PK1 cell monolayers expressing CLDN16, CLDN19, and their mutants, individually or in pairs, are shown.

on $\mathrm{P}_{\mathrm{Na}}$ or $\mathrm{P}_{\mathrm{Cl}}$ in either control or CLDN19-expressing cells, indicating a paracellular pathway for ion flux.

No profound effect of CLDN19 (with or without coexpression of CLDN16) was found on absolute $\mathrm{Mg}^{2+}$ permeability $\left(\mathrm{P}_{\mathrm{Mg}}\right.$ ) in LLC-PK1 cells (Supplemental Table 1). Interestingly, a small but significant suppression of $\mathrm{P}_{\mathrm{Mg}}$ was observed in CLDN19-expressing cells and in cells coexpressing CLDN16 and CLDN19 compared with control cells $(n=3, P<0.01)$.

We found no significant effects of CLDN19 on paracellular permeation of cation $\left(\mathrm{P}_{\mathrm{Na}}\right)$ or anion $\left(\mathrm{P}_{\mathrm{Cl}}\right)$ in MDCK cells, in contrast with the report by Angelow et al. (11). MDCK cells show high endogenous cation selectivity. In an earlier study (4), we were also unable to find significant effects of CLDN16 on either $\mathrm{P}_{\mathrm{Na}}$ or $\mathrm{P}_{\mathrm{Cl}}$ in MDCK cells. Thus, LLC-PK1 cells have proven to be a suitable model to determine CLDN16 and CLDN19 functions, as the functional analyses of CLDN16 mutations in LLC-PK1 cells are well correlated with the phenotypes of FHHNC patients (6).

FHHNC mutations in CLDN19. To study the physiological effects and trafficking patterns of human CLDN19 mutations, we expressed 3 previously published mutants (G20D, Q57E, and L90P; ref. 2) and a novel CLDN19 mutation (G123R; see Methods for patient information) in LLC-PK1 cells (Supplemental Figure 1B). Of the 4 mutations, 3 (G20D, L90P, and G123R) were in the transmembrane domains (Table 1), while Q57E was in the first extracellular loop. All of these mutations caused CLDN19 to lose its function significantly, reducing its ability to block $\mathrm{Cl}^{-}$permeation (Table 1). The mutations G20D and Q57E had trafficking defects: G20D was confined to the ER (Supplemental Figure 1C), consistent with previous findings (2), and Q57E adopted a diffuse pattern of localization throughout the apical membrane of polarized epithelia (Supplemental Figure 1C). Both mutants led to a complete loss of function, with $\mathrm{P}_{\mathrm{Na}} / \mathrm{P}_{\mathrm{Cl}}$ values close to baseline (G20D, $0.283 \pm 0.005$; Q57E, $0.387 \pm 0.015$; WT, $0.878 \pm 0.004 ; n=3$, $P<0.01$; Figure 1B and Table 1). The L90P and G123R mutants were localized in the TJ (Supplemental Figure 1C) and caused partial loss of function (Figure 1 and Table 1).

Synergistic effects of CLDN16 and CLDN19. Because the individual functions of both CLDN16 and CLDN19 were to increase the cation selectivity of the paracellular channels, we asked whether
CLDN16 and CLDN19 exert synergistic effects on ion selectivity when coexpressed in the TJ. As in our previous study (4), expression of CLDN16 alone in LLC-PK1 cells generated a large $\mathrm{Na}^{+}$flux $\left(\right.$CLDN16 $\mathrm{P}_{\mathrm{Na}}, 33.363 \pm 0.346 \times 10^{-6} \mathrm{~cm} / \mathrm{s}$; control $\mathrm{P}_{\mathrm{Na}}$, $\left.6.866 \pm 0.184 \times 10^{-6} \mathrm{~cm} / \mathrm{s} ; n=3, P<0.01\right)$ with no significant effects on $\mathrm{P}_{\mathrm{Cl}}$. Coexpression of CLDN16 and CLDN19 in LLC-PK1 cells resulted in a dramatic upregulation of $\mathrm{P}_{\mathrm{Na}}$ $\left(28.563 \pm 0.117 \times 10^{-6} \mathrm{~cm} / \mathrm{s}\right)$ and downregulation of $\mathrm{P}_{\mathrm{Cl}}$ $\left(7.425 \pm 0.118 \times 10^{-6} \mathrm{~cm} / \mathrm{s}\right)$, generating a highly cation-selective paracellular pathway $\left(\mathrm{P}_{\mathrm{Na}} / \mathrm{P}_{\mathrm{Cl}}, 3.849 \pm 0.076\right)$, as reflected by a large positive diffusion potential $(+8.80 \pm 0.10 \mathrm{mV})$ across the monolayer. The TJs in LLC-PK1 cells were transformed from having anion selectivity (at the LLC-PK1 cell baseline level) to having cation selectivity (at the LLC-PK1 synergistic level, resembling the MDCK cell baseline level; Figure 1A).

CLDN16 interacts with CLDN19 in yeast membranes. The synergistic effects of CLDN16 and CLDN19 on influencing ion selectivity of the TJ prompted us to ask whether CLDN16 physically interacts with CLDN19. CLDN1-CLDN3 interact side-to-side within the same cell, including both homomeric and heteromeric interactions, and interact selectively between cells in homotypic and heterotypic interactions (13). Biochemical characterization of claudins in insect cells suggests that claudins copolymerize to constitute TJ strand particles (each $\sim 10 \mathrm{~nm}$ in diameter, as revealed by freeze-fracture replicas) prior to their insertion into the plasma membrane (12). Deep-etch microscopy and subcellular fractionation expose a matrix of densely packed globular proteins on the cytoplasmic surfaces of the junctional membranes that is detergent resistant and sandwiched between the membrane surface and the actin cytoskeleton (17). The complex biochemical nature of the $\mathrm{TJ}$ and the strong protein-protein interactions within the TJ deny an unambiguous study of any selected claudin-claudin interaction within the matrix without interference from other proteins. Simple cell systems such as yeast cells or nonepithelial fibroblast cells do not form TJ and express no endogenous TJ proteins such as occludin or claudins. Thus, expressing tight junctional proteins such as CLDN16 and CLDN19 in these model systems allows the detection of possible direct protein-protein interactions independent of their crosslinking by other proteins within the TJ structure. 
A

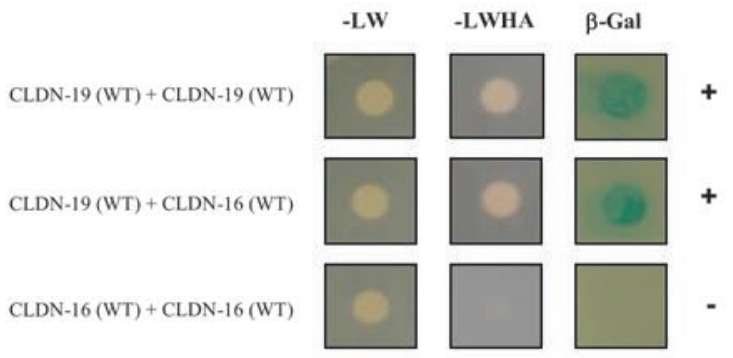

B

$-L W$

-LWHA
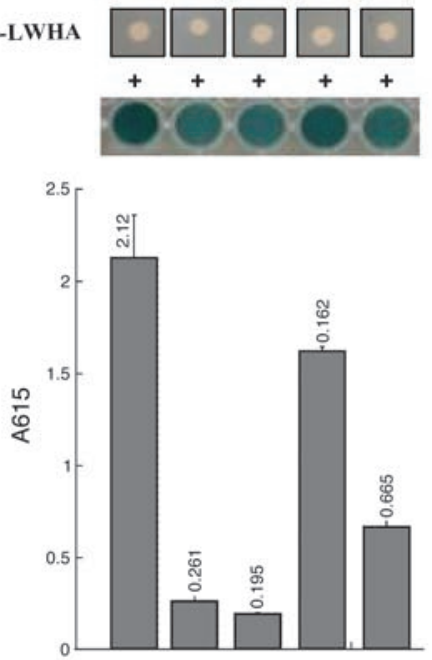

C

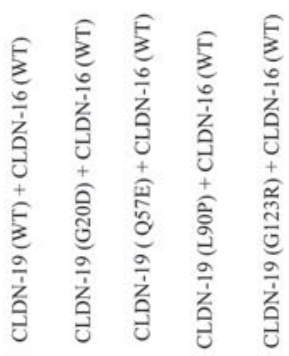

$-\mathrm{LW}$

-LWHA
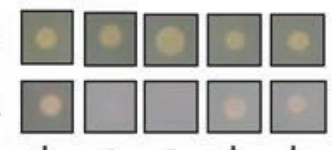

D

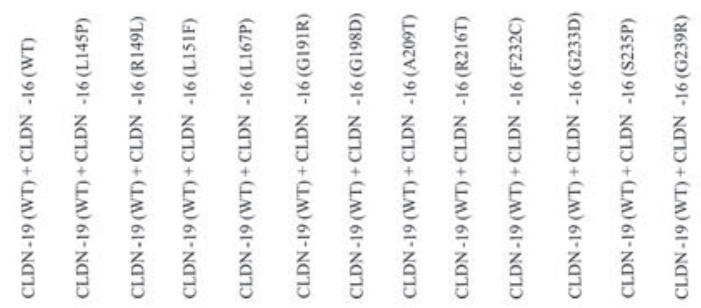

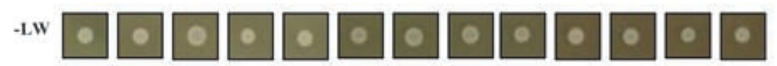
-LWHA $\square \square \square \square \square \square \square \square \square \square \square \square \square \square \square$
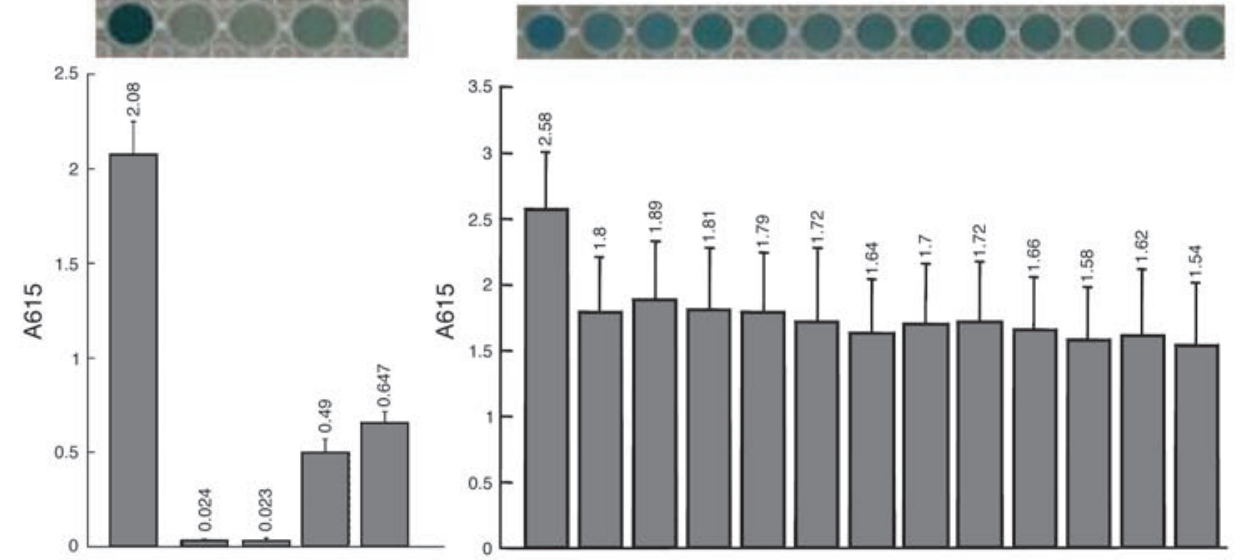

\section{Figure 2}

CLDN16 interacts with CLDN19 in yeast. (A) Y2H assays showing interaction of CLDN16-WT with CLDN19-WT and CLDN19-WT with CLDN19-WT, but not of CLDN16-WT with CLDN16-WT. Shown are plates with selective medium lacking leucine and tryptophan (-LW), indicating the transforming of both bait and prey vectors; with SD-LWHA, indicating the expression of reporter genes HIS3 and ADE2; and $\beta$-galactosidase assay (A615 values) for quantification of interaction strength. (B) Mutations in CLDN19 affecting its homomeric interaction. (C) Mutations in CLDN19 affecting its heteromeric interaction with CLDN16. (D) Mutations in CLDN16 affecting its heteromeric interaction with CLDN19.

To determine the homo- and heteromeric interactions between CLDN16 and CLDN19, we used the split-ubiquitin yeast 2-hybrid $(\mathrm{Y} 2 \mathrm{H})$ membrane protein interaction assay in Saccharomyces cerevisiae to examine interactions between claudins (see Methods and ref. 18). In the $\mathrm{Y} 2 \mathrm{H}$ membrane assay, a membrane protein of interest, the "bait," is fused to the C-terminal half of ubiquitin (Cub) along with an artificial transcriptional factor (TF). The putative interacting membrane proteins, called the "prey," are fused to the $\mathrm{N}$-terminal half of ubiquitin (Nub). Upon interaction of the 2 proteins, the reconstitution of ubiquitin $(\mathrm{Cub}+\mathrm{Nub})$ occurs. Ubiquitin is then recognized by ubiquitin-specific proteases, resulting in the cleavage of the TF. The released TF then enters the nucleus and activates transcription of the reporter genes HIS3, lacZ, and ADE2. Our data show that CLDN19 interacted with itself and with CLDN16, as assayed with all 3 reporters (HIS3, lacZ, and $A D E 2)$ in the yeast NMY51 strain (Figure 2A). As yeast cells do not make intercellular junctions and the reconstitution of ubiquitin $(\mathrm{Cub}+\mathrm{Nub})$ occurs within the same cell, these interactions between claudins are interpreted to be lateral associations within the membrane. The level of heteromeric interaction between CLDN19 and CLDN16 was equal to that of CLDN19 homomeric interaction, as quantified with the lacZ reporter (Figure 2, B and C). Intriguingly, CLDN16 failed to show interaction with itself on any of our 3 reporter assays (Figure $2 \mathrm{~A}$ ).

We coexpressed 4 missense mutations in CLDN19 and 12 missense mutations in CLDN16 that resulted in loss of function to document their influence on CLDN16/CLDN19 interaction. All mutations studied are known to cause the inherited human 
A

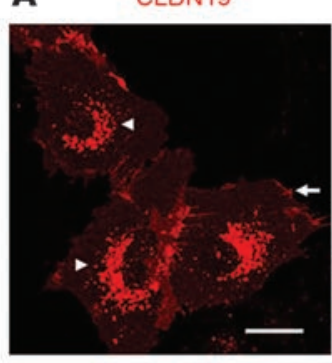

B
CLDN16

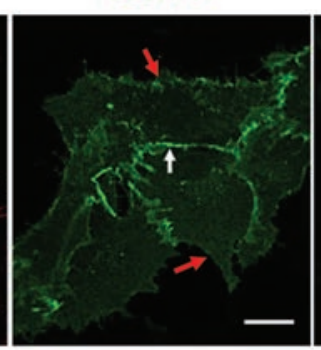

CLDN16

\begin{abstract}
co-localization
\end{abstract}
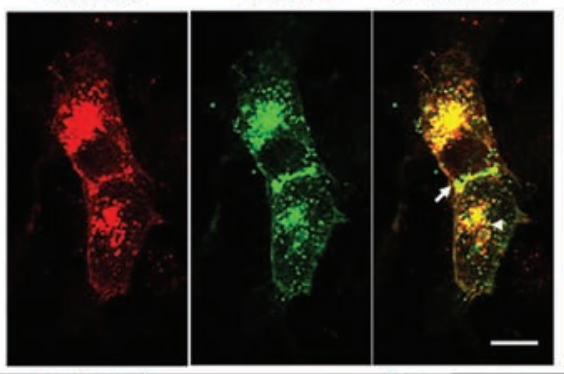

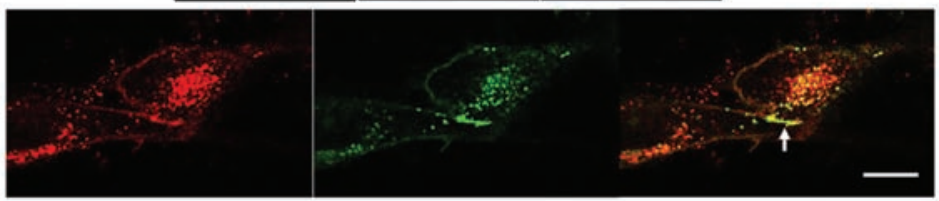

C

CLDN19

CLDN16-R149L

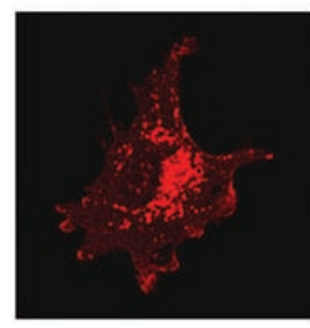

\begin{abstract}
co-localization
\end{abstract}

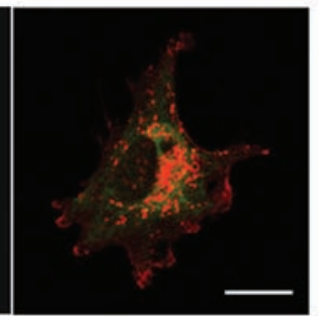

CLDN16-R149L

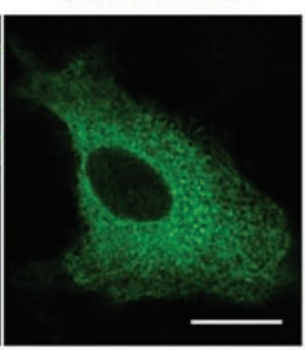

D

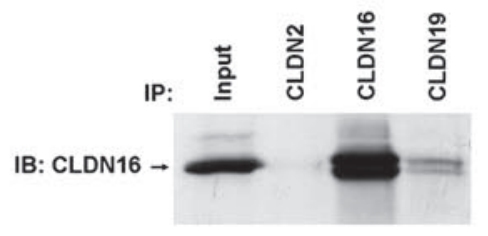

disease FHHNC. In the Y2H assay, 2 CLDN19 mutations (G20D and Q57E) resulted in a profound decrease in CLDN19 homomeric interactions ( $>90 \%, n=3, P<0.05$; Figure $2 \mathrm{~B}$ ) and the loss of heteromeric interaction with CLDN16 (close to $100 \%, n=3, P<0.05$; Figure 2C). The L90P and G123R mutations of CLDN19 mildly affected their homomeric interactions (Figure 2B) but severely affected their heteromeric interactions with CLDN16 $(>70 \%, n=3$, $P<0.05$; Figure 2C). On the other hand, all 12 CLDN16 mutations slightly, but not significantly, weakened their heteromeric interactions with CLDN19 (Figure 2D).

CLDN16 cotrafficks and coimmunoprecipitates with CLDN19 in epithelial cells. CLDN16 and CLDN19 localized to the TJ in both LLC-PK1 and MDCK monolayers (ref. 4 and Supplemental Figure 1). In subconfluent, not fully polarized LLC-PK1 cells, however, CLDN16 localized diffusely at sites of cell-cell interaction (Figure 3A, middle, white arrow) and variably at the non-

\section{Figure 3}

Cotrafficking and coimmunoprecipitation between CLDN16 and CLDN19 in unpolarized epithelial cells. (A) Confocal images showing subcellular localization of CLDN19, CLDN16, and CLDN16-R149L mutant in LLC-PK1 cells. CLDN19 localized to the plasma membrane (arrow) and the endosomes and lysosomes (arrowheads), CLDN16 localized to the plasma membrane (red arrows); and CLDN16-R149L was confined to the ER. In the middle panel, the white arrow denotes a site of cell-cell interaction. (B) Coexpression of CLDN16 with CLDN19 in LLC-PK1 cells altered the subcellular localization of CLDN16. Notably, CLDN16 was recruited to the endosomes and lysosomes (arrowhead), where colocalization with CLDN19 occurred. CLDN16 and CLDN19 colocalization was also found at the cell-cell interaction (arrow). (C) The confinement of CLDN16-R149L mutant to the ER was not affected by CLDN19 coexpression. No colocalization was found between CLDN16-R149L and CLDN19. (D) Coimmunoprecipitation of CLDN16 and CLDN19 cotransfected in HEK293 cells. Input lane shows $10 \%$ of input amount. Antibodies used for coimmunoprecipitation are shown above the lanes; antibody for blot visualization is shown at left. Scale bars: $10 \mu \mathrm{m}$. 


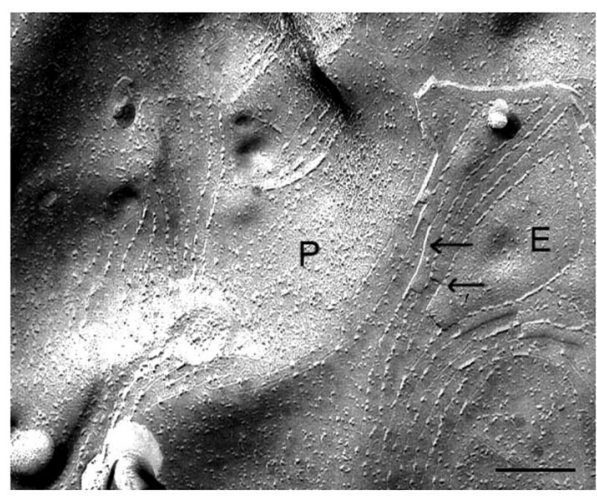

\section{Figure 4}

Freeze-fracture image of CLDN19-transfected L cells revealed TJ structures. Notably, a P-to-E fracture face transition was seen (arrows) where there was alignment of the TJ strands. Scale bar: $1 \mu \mathrm{m}$.

We also examined the localization patterns of CLDN16 and CLDN19 and the effects of CLDN19 on CLDN16 localization in MDCK and HEK293 cells and confirmed our findings in LLCPK1 cells (data not shown).

To directly document CLDN16/CLDN19 interaction, we attempted coimmunoprecipitation of CLDN16 and CLDN19 in sparsely plated HEK293 cells, an embryonic cell line with no TER. At low cell density, which minimizes cell-cell contacts and heterotypic interactions, interactions between CLDN16 and CLDN19 will be mostly heteromeric. Immunoblotting showed that antiCLDN19 antibody coimmunoprecipitated CLDN16 (Figure 3D), whereas anti-CLDN2 antibody (as a nonspecific binding control) had no effect. These biochemical data confirmed heteromeric interactions between CLDN16 and CLDN19 in epithelial cells.

CLDN16 and CLDN19 copolymerize into TJ strands. The findings of CLDN19 homomeric interaction and the lack of CLDN16 homomeric interaction in yeast were supported by studies of TJ strand formation in L fibroblasts. Freeze-fracture replicas revealed the assembly of $\mathrm{TJ}$ strands (homomeric interactions) in CLDN19-expressing L cells (Figure 4), but not in CLDN16expressing $\mathrm{L}$ cells (data not shown). In addition, the aligned fibrils and grooves seen at the transition between $\mathrm{P}$ and $\mathrm{E}$ fracture faces (Figure 4, arrows) indicated that CLDN19 was also capable of making homotypic interactions between adjacent cells. In L cells coexpressing CLDN16 and CLDN19, well-developed networks of TJ strands were observed, with the morphology similar to those of cells expressing CLDN19 alone. Immunogold labeling showed CLDN16 localization in these TJ strands of coexpressing cells, which also showed CLDN19 labeling (Figure 5). The lack of ability of CLDN16 to form strands on its own and its appearance in strands when coexpressed with CLDN19 indicated that CLDN19 recruited and copolymerized with CLDN16 into TJs, supporting the heteromeric interaction between CLDN16 and CLDN19. The heterotypic interaction between CLDN16 and CLDN19 is discussed below.

Disruption of CLDN16 and CLDN19 interaction abolishes their synergistic effects. Genetic analysis has linked distinct missense mutations in CLDN16 and CLDN19 to FHHNC $(2,6,19)$. We have presented a detailed characterization of both CLDN16 mutations (4, 6) and CLDN19 mutations (present study). We have also shown that when CLDN16-WT was expressed with CLDN19-WT, a syn- ergistic effect on ion selectivity was found, transforming the TJs of LLC-PK1 cells from anion selectivity to cation selectivity $\left(\mathrm{P}_{\mathrm{Na}} / \mathrm{P}_{\mathrm{Cl}}\right.$, $3.849 \pm 0.076$; Figure $1 \mathrm{~B}$ and Table 1), whereas both CLDN16 and CLDN19 alone merely reduced the anion selectivity of the TJ and rendered the $\mathrm{P}_{\mathrm{Na}} / \mathrm{P}_{\mathrm{Cl}}$ ratio close to 1 (Figure $1 \mathrm{~B}$ and Table 1 ). We next asked whether this synergistic effect could still be found when CLDN19-WT was mixed with CLDN16 mutants or CLDN16-WT mixed with CLDN19 mutants. To allow CLDN16/ CLDN19 interaction to occur in the TJ, we avoided choosing mutations in CLDN16 or CLDN19 that had trafficking defects. Five CLDN16 mutants (L145P, L151F, G191R, A209T, and F232C) that localized to the TJ when coexpressed with CLDN19-WT failed to elicit any synergistic effect. The dilution potential values of these coexpressions were close to that of CLDN19-WT expression alone (Figure 1A). The interaction strength between these CLDN16 mutants and CLDN19-WT was notably reduced (assayed in yeast; Figure 2D). CLDN19 mutants L90P and G123R, which localized to the TJ when coexpressed with CLDN16-WT, also showed no synergistic effects. The dilution potential values of CLDN19-L90P+CLDN16-WT and CLDN19-G123R+CLDN16WT were similar to that of CLDN16-WT alone (Figure 1A). The interaction strength between these CLDN19 mutants and CLDN16-WT was also reduced (Figure 2C). Therefore, decreases in CLDN16-CLDN19 interactions, caused by mutations in CLDN16 or CLDN19, provided an explanation for the abolishing of CLDN16-CLDN19 synergistic effects.

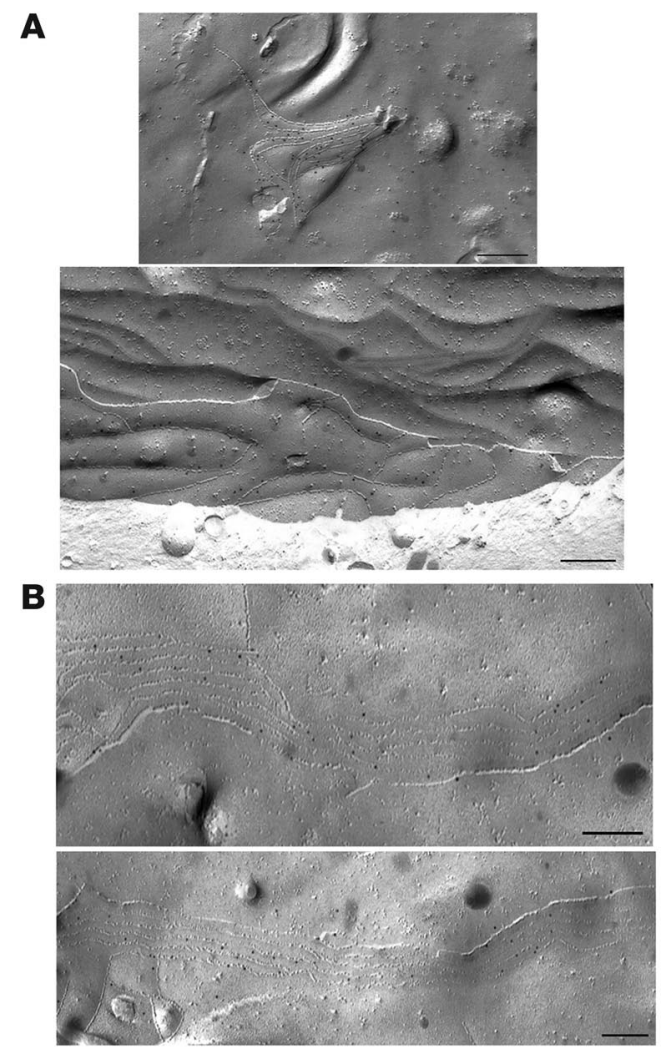

\section{Figure 5}

Freeze-fracture images of CLDN19/CLDN16 cotransfected L cells with immunogold (10 nm gold particle) labeling for CLDN19 and CLDN16. Note the localization of CLDN19 (A) and CLDN16 (B) within the welldeveloped networks of TJ strands. Scale bars: $200 \mathrm{~nm}$. 
Table 2

Paracellular ion conductance in LLC-PK1 cells expressing CLDN16, CLDN19, and both combined

\begin{tabular}{lccrrr} 
Construct & TER $\left(\Omega \cdot \mathbf{c m}^{2}\right)$ & Dilution potential $(\mathbf{m V})$ & $\mathbf{P}_{\text {Na }} / \mathbf{P}_{\mathrm{Cl}}$ & $\mathbf{P}_{\text {Na }}(\mathbf{1 0}-\mathbf{6} \mathbf{c m} / \mathbf{s})$ & $\mathbf{P}_{\mathbf{C l}}\left(\mathbf{1 0} 0^{-6} \mathbf{c m} / \mathbf{s}\right)$ \\
Vector & $61.0 \pm 2.1$ & $-8.13 \pm 0.19$ & $0.296 \pm 0.010$ & $6.866 \pm 0.184$ & $23.220 \pm 0.182$ \\
CLDN16 & $33.0 \pm 1.5$ & $2.97 \pm 0.19$ & $1.500 \pm 0.038$ & $33.363 \pm 0.346$ & $22.257 \pm 0.346$ \\
CLDN19 & $143.3 \pm 1.9$ & $-0.97 \pm 0.03$ & $0.878 \pm 0.004$ & $5.999 \pm 0.014$ & $6.836 \pm 0.014$ \\
CLDN16+CLDN19 coexpression & $50.7 \pm 3.7$ & $8.80 \pm 0.10$ & $3.849 \pm 0.076$ & $28.563 \pm 0.117$ & $7.425 \pm 0.118$ \\
CLDN16+CLDN19 coculture (1:1 mixing ratio) & $64.3 \pm 1.9$ & $-3.23 \pm 0.09$ & $0.643 \pm 0.008$ & $11.220 \pm 0.085$ & $17.460 \pm 0.085$ \\
CLDN16+CLDN19 coculture (1:2 mixing ratio) & $82.3 \pm 0.9$ & $-5.10 \pm 0.15$ & $0.490 \pm 0.011$ & $7.357 \pm 0.114$ & $15.027 \pm 0.113$ \\
CLDN16+CLDN19 coculture (2:1 mixing ratio) & $56.0 \pm 1.5$ & $-1.87 \pm 0.07$ & $0.776 \pm 0.007$ & $14.327 \pm 0.073$ & $18.447 \pm 0.076$ \\
\hline
\end{tabular}

\section{Discussion}

Our data show that CLDN16 and CLDN19, 2 proteins that are required for renal reabsorption of $\mathrm{Mg}^{2+}$, interacted in cell membranes. The interaction was supported by 4 independent lines of evidence: (a) a positive $\mathrm{Y} 2 \mathrm{H}$ assay; (b) cotrafficking and colocalization in epithelial cells; (c) coimmunoprecipitation in epithelial cells; and (d) functional synergistic effects on increasing the cation selectivity of TJs.

Claudin interaction affecting its function. The synergistic effects of CLDN16 and CLDN19 on the ion selectivity of TJs resulted from their interaction. We have found copolymerization (heteromeric interaction) between CLDN16 and CLDN19 within the cell membrane using yeast and epithelial cell models. The lack of CLDN16 homomeric interaction and its dependence upon CLDN19 to be recruited and copolymerize into TJ strands emphasize the importance of CLDN16/ CLDN19 heteromeric interaction in the trafficking and polymerizing of CLDN16, although the function of CLDN16 may not depend upon CLDN19.

We have also tested heterotypic (headto-head) interaction between CLDN16 and CLDN19 using the confocal immunofluorescent imaging method developed by Furuse et al. (13). When L fibroblast cells expressing CLDN16 were cocultured with L cells expressing CLDN19, no colocalization of CLDN16 and CLDN19 was found at areas of cell-cell contact (Supplemental Figure 2), suggesting a lack of heterotypic interaction. The lack of heterotypic interaction was further supported by a functional analysis. When LLC-PK1 cells expressing CLDN16 were cocultured with LLC-PK1 cells expressing CDLN19, the cation selectivity of TJs were not as high as that of cells expressing either CLDN16 or CLDN19 alone, as reflected by the more negative dilution potential values $(n=3$, $P<0.01$; Table 2).

When CLDN16 and CLDN19 were coexpressed in LLC-PK1 cells, ion selectivity of the paracellular pathway was dramatically converted from anion to cation selective (Table 1). Mutations in either claudin that interfered with their ability to interact disrupted the synergistic physiological change.
Taken together, these data indicate that CLDN16 and CLDN19 associated through heteromeric interactions and that this association changed the selectivity of the paracellular channels. Certain CLDN16 (L145P, L151F, G191R, A209T, and F232C) or CLDN19 (L90P and G123R) mutations that cause FHHNC interfered with CLDN16/CLDN19 interaction. The same mutations also abolished the synergistic physiological effects of the pair.

A limitation of this study is that the biochemical interaction between CLDN16 and CLDN19 has been studied in yeast and L cells, while the physiological data were taken from LLC-PK1 epithelial monolayers. It is known that the process of junctional assembly differs between L cells and polarized epithelia (16), and that junctions do not form in yeast. Nevertheless, the assembly of multimeric proteins in eukaryotes is generally carried out in the

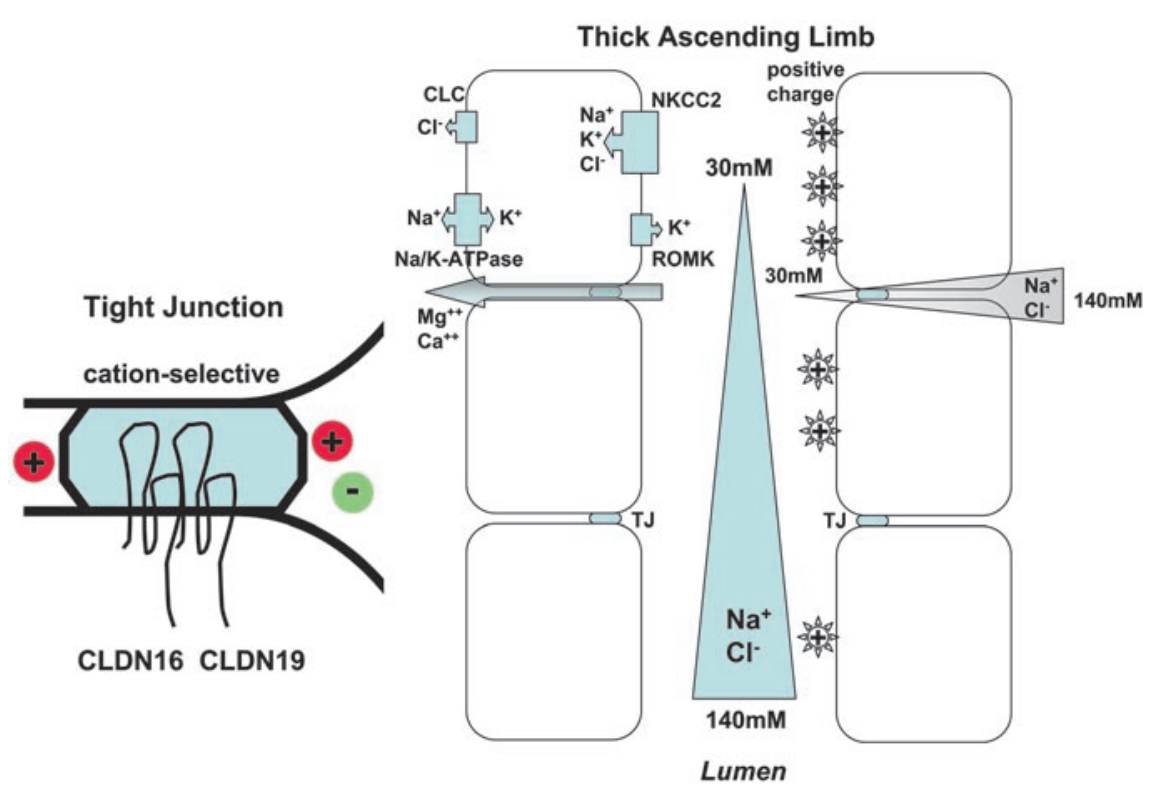

Figure 6

Function of $\mathrm{TJ}$ and mechanism of $\mathrm{Mg}^{2+}$ reabsorption. In $\mathrm{TAL}, \mathrm{NaCl}$ reabsorption is mediated through $\mathrm{Na}^{+} \mathrm{K}^{+} \mathrm{Cl}^{-}$-cotransporter type 2 (NKCC2) in the apical membrane. On the basolateral side, $\mathrm{Na} / \mathrm{K}-\mathrm{ATP}$ ase provides the energy source and also allows $\mathrm{Na}^{+}$to exit the cell in exchange for $\mathrm{K}^{+}$entry. $\mathrm{K}^{+}$is secreted to the lumen side via the renal outer medullary $\mathrm{K}^{+}$channel (ROMK). $\mathrm{Cl}^{-}$exits the cell via the $\mathrm{Cl}^{-}$channel $(\mathrm{CLC})$. Continuous $\mathrm{NaCl}$ reabsorption along $\mathrm{TAL}$ results in gradual tubular fluid dilution and the development of a transepithelial $\mathrm{NaCl}$ concentration gradient (from peritubular space, $140 \mathrm{mM}$, down to lumen, $30 \mathrm{mM}$ ). As the epithelial cells in TAL are joined by cation-selective $\mathrm{TJ}$, the $\mathrm{NaCl}$ concentration gradient results in a lumen-positive transepithelial diffusion potential. This positive potential drives the reabsorption of $\mathrm{Mg}^{2+}$ and $\mathrm{Ca}^{2+}$ through the TJ (known as the paracellular pathway). CLDN16 and CLDN19 interact in TJs and contribute to the cation selectivity of TJs. 
ER (20) or in the Golgi apparatus (21), so the associations observed between CLDN16 and CLDN19 likely occur in all cell types.

CLDN16 and CLDN19 in pathogenesis of FHHNC. The reabsorption of $\mathrm{Mg}^{2+}$ in the TAL occurs via the paracellular pathway and is highly dependent on the transepithelial potential as a driving force (22). The potential is generated by the electrogenic $\mathrm{NaCl}$ reabsorption resulting in tubular fluid dilution and the development of a transepithelial $\mathrm{NaCl}$ concentration gradient (from peritubular space down to lumen). As the epithelial cells in TAL are joined by cation-selective $\mathrm{TJ}$ s, the $\mathrm{NaCl}$ concentration gradient results in a lumen-positive transepithelial diffusion potential, the driving force for $\mathrm{Mg}^{2+}$ reabsorption (Figure 6). Both CLDN16 and CLDN19 contribute to the cation selectivity of TJs. CLDN16 functions as a $\mathrm{Na}^{+}$channel (4), whereas CLDN19 functions as a $\mathrm{Cl}^{-}$blocker. When TJs of in vitro cultured cell models (lacking endogenous CLDN16 and CLDN19) were supplemented with both CLDN16 and CLDN19, they show high cation selectivity, owing to the synergistic effects of the CLDN16/CLDN19 protein complex. $\mathrm{P}_{\mathrm{Na}} / \mathrm{P}_{\mathrm{Cl}}$ was estimated to be $3.849 \pm 0.076$ (Table 1 ), very close to the $\mathrm{P}_{\mathrm{Na}} / \mathrm{P}_{\mathrm{Cl}}$ of native TAL of the mouse kidney, previously reported to be $3.1 \pm 0.3(7)$. Depleting CLDN16 generated TJs with reduced cation selectivity (in vitro $\mathrm{P}_{\mathrm{Na}} / \mathrm{P}_{\mathrm{Cl}}, 0.878 \pm 0.004$; Table 1 ; in vivo $\mathrm{P}_{\mathrm{Na}} / \mathrm{P}_{\mathrm{Cl}}, 1.5 \pm 0.1$; ref. 7 ), leading to decreases in the lumen-positive diffusion potential. We are presently studying to determine how depleting CLDN19 in vivo affects TJ functions. Human FHHNC mutations in CLDN16 or CLDN19 that abolish the CLDN16/CLDN19 synergistic effects generate tight junctional cation selectivity close to that of the CLDN16-null or CLDN19null backgrounds, readily explaining the devastating FHHNC phenotypes in human patients $(1,2,6,19)$. When both CLDN16 and CLDN19 are lost in the kidney, TJs will become highly permeable to $\mathrm{Cl}^{-}$but not to $\mathrm{Na}^{+}$and show high anion selectivity, while reversing the lumen potential to negative values. Therefore, no $\mathrm{Mg}^{2+}$ can be reabsorbed. In addition, more $\mathrm{Cl}^{-}$and less $\mathrm{Na}^{+}$will flow back to the lumen side from peritubular space. More luminal $\mathrm{Cl}^{-}$will also disrupt the electric neutrality of tubular fluid, leading to retention of more $\mathrm{Na}^{+}$and $\mathrm{K}^{+}$to balance the excess of $\mathrm{Cl}^{-}$.

Other claudins expressed in TAL, including CLDN3, CLDN10, and CLDN11, may also play important roles in control of the ion selectivity of the TJ (23). In particular, CLDN10 decreases the cation selectivity of the TJ in both MDCK cells and LLC-PK1 cells (24), thus making it a good candidate as the negative regulator of the CLDN16/CLDN19 functional complex.

\section{Methods}

Antibodies and cell lines. The following antibodies were used in this study: rabbit polyclonal anti-CLDN1, anti-CLDN2, anti-CLDN3, anti-CLDN7, anti-CLDN16 and mouse monoclonal anti-CLDN4 and anti-occludin (Zymed Laboratories); rabbit anti-CLDN19 (a kind gift from M. Furuse, Kobe University Graduate School of Medicine, Kobe, Japan); fluorescein isothiocyanate-labeled goat anti-rabbit immunoglobulin $\mathrm{G}$ and rhodamine-labeled goat anti-mouse immunoglobulin $\mathrm{G}$ (Chemicon); horseradish peroxidase-labeled donkey anti-rabbit and anti-mouse immunoglobulin G (Amersham Pharmacia Biotech). MDCK II cells and LLC-PK1 cells were cultured in MEM (Invitrogen) supplemented with 10\% FBS and penicillin/ streptomycin; mouse L cells and HEK293 cells in DMEM supplemented with $10 \%$ FBS, penicillin/streptomycin, and $1 \mathrm{mM}$ sodium pyruvate.

Molecular cloning and retrovirus production. The following full-length mammalian claudins were cloned into the retroviral vector PQCXIN (gift from J. Brugge, Harvard Medical School): human CLDN16 (GenBank accession no. AF152101) and human CLDN19 (GenBank accession no. BC030524). The site-directed mutagenesis was performed with a PCR-based mutagenesis method (Stratagene). Molecular clones for each of the mutants were verified by DNA sequencing. VSV-G pseudotyped retroviruses were produced in HEK293 cells and used to infect MDCK cells at a titer of $1 \times 10^{6}$ $\mathrm{CFU} / \mathrm{ml}$, as described previously (4).

Protein electrophoresis and immunoblotting. Confluent cells were dissolved in lysis buffer (50 mM Tris- $\mathrm{HCl}, \mathrm{pH} 7.5 ; 150 \mathrm{mM} \mathrm{NaCl}$; $1 \%$ SDS; and protease inhibitor cocktail; Pierce). After shearing with a 23-gauge needle, lysates (containing $20 \mu \mathrm{g}$ total protein) were subjected to SDS-PAGE under denaturing conditions and transferred to a nitrocellulose membrane, followed by blocking with $3 \%$ nonfat milk, incubation with primary antibodies (diluted 1:1,000) and horseradish peroxidase-labeled secondary antibody (diluted 1:5,000), and exposure to an ECL Hyperfilm (Amersham). Molecular mass was determined relative to protein markers (BioRad).

Coimmunoprecipitation. HEK293 cells expressing both CLDN16 and CLDN19 were lysed in $50 \mathrm{mM}$ Tris ( $\mathrm{pH}$ 8.0) by $25-30$ repeated passages through a 25 -gauge needle, followed by centrifugation at $5,000 \mathrm{~g}$. The membranes of lysed cells were extracted using CSK buffer $(150 \mathrm{mM}$ $\mathrm{NaCl} ; 1 \%$ Triton X-100; $50 \mathrm{mM}$ Tris, $\mathrm{pH}$ 8.0; and protease inhibitors). The membrane extract was precleared by incubation with protein A/G-sepharose (Sigma-Aldrich) prior to coimmunoprecipitation. The precleared membrane extract was incubated for $16 \mathrm{~h}$ at $4^{\circ} \mathrm{C}$ with anti-CLDN2, antiCLDN16, and anti-CLDN19 antibodies. Antibody-bound material was pelleted with protein $\mathrm{A} / \mathrm{G}$-sepharose, washed 3 times with CSK buffer, and detected by immunoblotting.

Immunolabeling and confocal microscopy. Cells grown on coverslips were fixed with $4 \%$ paraformaldehyde in $0.1 \mathrm{M} \mathrm{PBS}$; cells grown on Transwell inserts (Corning) were fixed with cold methanol at $-20^{\circ} \mathrm{C}$, followed by blocking with PBS containing 10\% FBS and incubation with primary antibodies (diluted 1:300) and FITC- or rhodamine-labeled secondary antibodies (diluted 1:200). After washing with PBS, slides were mounted with Mowiol (CalBiochem). Confocal analyses were performed using the Nikon TE2000 confocal microscopy system equipped with Plan-Neofluar $\times 40$ (NA 1.3 oil) and $\times 63$ (NA 1.4 oil) objectives and krypton-argon laser (488 and 543 lines). For the dual imaging of FITC and rhodamine, fluorescent images were collected by exciting the fluorophores at $488 \mathrm{~nm}$ (FITC) and $543 \mathrm{~nm}$ (rhodamine) with argon and HeNe lasers, respectively. Emissions from FITC and rhodamine were detected with the bandpass FITC filter set of 500-550 $\mathrm{nm}$ and the long-pass rhodamine filter set of $560 \mathrm{~nm}$, respectively. All images were converted to JPEG format and arranged using Photoshop 6.0 (Adobe).

Freeze fracture. Confluent monolayers of mouse L cells individually expressing or coexpressing CLDN16 and CLDN19, or confluent monolayers in which $\mathrm{L}$ cells expressing CLDN16 were cocultured with L cells expressing CLDN19, were fixed in 2\% glutaraldehyde in Dulbecco PBS (DPBS) for 20 minutes at $4^{\circ} \mathrm{C}$. They were rinsed twice in DPBS, scraped from the substrate, and infiltrated with $10 \%, 20 \%$, and $25 \%$ glycerol in $0.1 \mathrm{M}$ cacodylate buffer, $\mathrm{pH} 7.3$, over $1 \mathrm{~h}$ at $4{ }^{\circ} \mathrm{C}$. Cell pellets were frozen in liquid nitrogen slush and freeze fractured at $-115^{\circ} \mathrm{C}$ in a Balzers 400 freeze-fracture unit. After cleaning with sodium hypochlorite, replicas were examined by electron microscopy at a magnification of $\times 62,500$.

Freeze fracture immunolabeling. The method for immunogold labeling was described previously (25). Briefly, the monolayers were fixed in $1 \%$ paraformaldehyde in DPBS for 15 minutes at $4{ }^{\circ} \mathrm{C}$. They were rinsed 3 times in DPBS, scraped from the substrate, and infiltrated with $10 \%, 20 \%$, and $25 \%$ glycerol in $0.1 \mathrm{M}$ cacodylate buffer over $1 \mathrm{~h}$ at $4^{\circ} \mathrm{C}$. Cell pellets were frozen in liquid nitrogen slush and freeze fractured at $-115^{\circ} \mathrm{C}$. The replicas were cleaned by floating them on the surface of $2.5 \% \mathrm{SDS}, 30 \mathrm{mM}$ sucrose in $10 \mathrm{mM}$ Tris- $\mathrm{HCl}(\mathrm{pH} 8.3)$ using a magnetic stir plate for $2 \mathrm{~h}$ at room temperature. 
The replicas were rinsed in 4 changes of DPBS over $1 \mathrm{~h}$ and incubated with $5 \%$ ovalbumin in DPBS for $30 \mathrm{~min}$. They were then incubated with rabbit anti-CLDN16 or anti-CLDN19 (diluted 1:100 in blocking solution) for $1 \mathrm{~h}$ at room temperature. After replicas were rinsed in DPBS, they were incubated for $1 \mathrm{~h}$ with protein A gold $(10 \mathrm{~nm})$ diluted 1:100 in ovalbumin/DPBS. After rinsing in DPBS, the labeled replicas were fixed with $0.5 \%$ glutaraldehyde in DPBS and picked up on Formvar-coated copper grids. Replicas were examined with a Philips 301 electron microscope.

Clinical analysis of identifying novel CLDN19 mutations. CLDN19 mutation analysis was performed by direct sequencing. In brief, an overlapping set of primers amplifying the human CLDN19 coding sequences and the intron/ exon boundaries was used, as described previously (2). Bidirectional direct sequencing was performed using the BigDye Terminator Cycle Sequencing Kit V.1.1 (Applied Biosystems) on an ABI 3100 capillary sequencer. The absence of both CLDN19 mutations in unaffected individuals was confirmed by the analysis of 196 ethnically matched control chromosomes. The male infant patient in which the novel CLDN19 mutation was identified was the first child of healthy parents who were second-degree relatives of Arab origin. At 3 months of age, bilateral medullary nephrocalcinosis was detected. Clinical analyses revealed excessive hypercalciuria $\left(\mathrm{Ca}^{++} /\right.$creatinine ratio $\left.11[\mathrm{~mol} / \mathrm{mol}]\right)$ and hypomagnesemia $(0.43 \mathrm{mmol} / \mathrm{l})$, fulfilling the diagnostic criteria for FHHNC. The infant had a normal glomerular filtration rate, no evidence for urinary tract infections, no neurological symptoms of hypomagnesemia, and no nystagmus. Ophthalmologic examination revealed myopia, but no evidence for macular coloboma was found at the age of 8 months. Under magnesium supplementation, the clinical course was uneventful to 20 months, the patient's age at the conclusion of the study. An uncle from the paternal side and 1 first-degree cousin had nephrolithiasis. Renal ultrasound in both parents was normal. Direct sequencing revealed a homozygous missense mutation (G123R) in CLDN19 from this patient.

$\mathrm{Y} 2 \mathrm{H}$ membrane protein interaction assay. $\mathrm{Y} 2 \mathrm{H}$ membrane protein interaction assay (MoBiTec Molecular Biotechnology) was used for analyzing the specific interactions among CLDN16, CLDN19, and their mutants. The DNA fragments were amplified using PCR and cloned into the vectors pBT3-C and PBT3-N, such that they were in frame with the Cub-TF cassette placed downstream and upstream, respectively (for bait vectors with Cub fusion), and similarly into the vectors PPR3-C and pPR3-N (for prey vectors with $\mathrm{Nub}$ fusion). The assay was performed by transforming the yeast strain NMY51 with $1.5 \mu \mathrm{g}$ bait vectors. The correct expression of the bait vectors for CLDN16 and CLDN19 was verified by Western blot using LexA mouse monoclonal antibody (Santa Cruz Biotechnology). Verification of correct topology of all the baits was performed using pAI-Alg5 and pDL2-Alg5 control preys, and the upper limit of selection stringency of the baits was determined using selective triple dropout medium lacking leucine, tryptophan, histidine, and adenine (SD-LWHA). Following this, the yeast strains expressing the bait proteins were transformed with $1.5 \mu \mathrm{g}$ prey vectors. Transformed yeast cells were plated on selective dropout media lacking leucine and tryptophan and incubated for growth of positive transformants. Next, 3-6 independent positive transformants were selected and resuspended in $50 \mathrm{ml} 0.9 \% \mathrm{NaCl}$ buffer; $5 \mu \mathrm{l}$ of each suspension was spotted on SD-LWHA medium. Growth of colonies on the selective medium was scored as positive for interaction. To further verify the positive interactions, $\beta$-galactosidase activity was performed following the manufacturer's protocols (MoBiTec $\mathrm{GmbH}$ ). To determine the strength of interaction, colonies from SD-LWHA plates were respotted on SD-LWHA plates supplemented with varying amounts of 3-amino-1,2,4-triazole (3AT; $10,20,40$ and $60 \mathrm{mM})$. Quantitative measurements of $\beta$-galactosidase activity was performed by lysing $1 \mathrm{ml}$ of 2 OD units each of the overnight culture with $0.05 \mathrm{M}$ Tris, $1 \%$ SDS ( $\mathrm{pH} 8.8$ ), and $\sim 100 \mu$ lacid-washed glass beads (Sigma-Aldrich), followed by 3 freeze-thaw cycles in liquid nitrogen. The lysate was incubated for 30 minutes with $10 \mu \mathrm{l}$ 10\% X-Gal (Carl Roth). Color development was measured using a spectrophotometer and scored as an indicator of the strength of the interaction. Blank measurements were performed with untransformed yeast cells.

Electrophysiological measurements. Electrophysiological studies were performed on cell monolayers grown on porous filters (Transwell) as previously described (4). Voltage and current clamps were performed using the EVC4000 Precision V/I Clamp (World Precision Instruments) with $\mathrm{Ag} / \mathrm{AgCl}$ electrodes and an agarose bridge containing $3 \mathrm{M} \mathrm{KCl}$. TER was measured using the Millicell-ERS and chopstick electrodes (Millipore). TER of the confluent monolayer of cells was determined in buffer A $(145 \mathrm{mM} \mathrm{NaCl}$, $2 \mathrm{mM} \mathrm{CaCl}_{2}, 1 \mathrm{mM} \mathrm{MgCl}_{2}, 10 \mathrm{mM}$ glucose, and $10 \mathrm{mM}$ HEPES, $\mathrm{pH} 7.4$ ), and the TER of blank filters was subtracted. Dilution potentials were measured when buffer B ( $80 \mathrm{mM} \mathrm{NaCl}, 130 \mathrm{mM}$ mannitol, $2 \mathrm{mM} \mathrm{CaCl}_{2}, 1 \mathrm{mM}$ $\mathrm{MgCl}_{2}, 10 \mathrm{mM}$ glucose, and $10 \mathrm{mM}$ HEPES, $\mathrm{pH}$ 7.4) replaced buffer A on the apical or basal side of filters. Electrical potentials obtained from blank inserts were subtracted from those obtained from inserts with confluent growth of cells. $\mathrm{P}_{\mathrm{Na}} / \mathrm{P}_{\mathrm{Cl}}$ for the monolayer was calculated from the dilution potential using the Goldman-Hodgkin-Katz equation. $\mathrm{P}_{\mathrm{Na}}$ and $\mathrm{P}_{\mathrm{Cl}}$ were calculated using the Kimizuka-Koketsu equation. $\mathrm{P}_{\mathrm{Mg}}$ across monolayers was determined as described previously (4).

Statistics. The significance of differences between groups was tested by ANOVA (Statistica 6.0; Statsoft 2003). When the all-effects $F$ value was significant $(P<0.05)$, post-hoc analysis of differences between individual groups was made with the Neuman-Keuls test. Values were expressed as mean \pm SEM unless otherwise stated.

\section{Acknowledgments}

We thank Joan Brugge (Harvard Medical School) and Mikio Furuse (Kobe University, Kobe, Japan) for kindly providing reagents. We thank B. Hoppe (University Children's Hospital, Cologne, Germany) for DNA and clinical information of the new FHHNC patient. We also thank the Nikon Imaging Centre of Harvard Medical School for its excellent assistance on confocal microscopy. This work was supported by NIH grants EY02430, GM37751, HL25822, HL36781, and EY014127; by the Deutsche Forschungsgemeischaft; by the Kempkes Foundation of the University of Marburg; and by the Rhoen AG.

Received for publication September 18, 2007, and accepted in revised form November 7, 2007.

Address correspondence to: Daniel A. Goodenough, Department of Cell Biology, Harvard Medical School, 240 Longwood Avenue, Boston, Massachusetts 02115, USA. Phone: (617) 432-1652; Fax: (617) 432-2955; E-mail: daniel_goodenough@hms.harvard.edu.

Jianghui Hou and Aparna Renigunta contributed equally to this work.
1. Simon, D.B., et al. 1999. Paracellin-1, a renal tight junction protein required for paracellular $\mathrm{Mg}^{2+}$ resorption. Science. 285:103-106.

2. Konrad, M., et al. 2006. Mutations in the tightjunction gene claudin 19 (CLDN19) are associ- ated with renal magnesium wasting, renal failure, and severe ocular involvement. Am. J. Hum. Genet. 79:949-957.

3. Anderson, J.M., Van Itallie, C.M., and Fanning, A.S. 2004. Setting up a selective barrier at the apical junction complex. Curr. Opin. Cell Biol. 16:140-145. 4. Hou, J., Paul, D.L., and Goodenough, D.A. 2005. Paracellin-1 and the modulation of ion selectivity of tight junctions. J. Cell Sci. 118:5109-5118.

5. Hou,J., Gomes, A.S., Paul, D.L., and Goodenough, D.A. 
2006. Study of claudin function by RNA interference. J. Biol. Chem. 281:36117-36123.

6. Konrad, M., et al. 2008. CLDN16 genotype predicts renal decline in familial hypomagnesemia with hypercalciuria and nephrocalcinosis. J. Am. Soc. Nephrol. 19:171-181.

7. Hou, J., et al. 2007. Transgenic RNAi depletion of claudin-16 and the renal handling of magnesium. J. Biol. Chem. 282:17114-17122.

8. Miyamoto, T., et al. 2005. Tight junctions in Schwann cells of peripheral myelinated axons: a lesson from claudin-19-deficient mice. J. Cell Biol. 169:527-538

9. Luk, J.M., et al. 2004. Sp1 site is crucial for the mouse claudin-19 gene expression in the kidney cells. FEBS Lett. 578:251-256.

10. Lee, N.P., et al. 2006. Kidney claudin-19: localization in distal tubules and collecting ducts and dysregulation in polycystic renal disease. FEBS Lett. 580:923-931.

11. Angelow, S., El-Husseini, R., Kanzawa, S.A., and Yu, A.S. 2007. Renal localization and function of the tight junction protein, claudin-19. Am. J. Physiol. Renal Physiol. 293:F166-F177.

12. Mitic, L.L., Unger, V.M., and Anderson, J.M. 2003. Expression, solubilization, and biochemical characterization of the tight junction transmembrane protein claudin-4. Protein Sci. 12:218-227.

13. Furuse, M., Sasaki, H., and Tsukita, S. 1999. Manner of interaction of heterogeneous claudin species within and between tight junction strands. J. Cell Biol. 147:891-903.

14. Stevenson, B.R., Siliciano, J.D., Mooseker, M.S., and Goodenough, D.A. 1986. Identification of ZO-1: a high molecular weight polypeptide associated with the tight junction (zonula occludens) in a variety of epithelia. J. Cell Biol. 103:755-766.

15. Gumbiner, B., Lowenkopf, T., and Apatira, D. 1991. Identification of a $160 \mathrm{kDa}$ polypeptide that binds to the tight junction protein ZO-1. Proc. Natl. Acad. Sci. U. S. A. 88:3460-3464.

16. Umeda, K., et al. 2006. ZO-1 and ZO-2 independently determine where claudins are polymerized in tight-junction strand formation. Cell. 126:741-754.

17. Stevenson, B.R., and Goodenough, D.A. 1984 Zonulae occludentes in junctional complexenriched fractions from mouse liver: preliminary morphological and biochemical characterization. J. Cell Biol. 98:1209-1221.

18. Iyer, K., et al. 2005. Utilizing the split-ubiquitin membrane yeast two-hybrid system to identify protein-protein interactions of integral membrane proteins. Sci. STKE. 2005:pl3.

19. Weber, S., et al. 2001. Novel paracellin-1 mutations in 25 families with familial hypomagnesemia with hypercalciuria and nephrocalcinosis. J. Am. Soc. Nephrol. 12:1872-1881.

20. Hurtley, S.M., and Helenius, A. 1989. Protein oligomerization in the endoplasmic reticulum. Annu. Rev. Cell Biol. 5:277-307.

21. Musil, L.S., and Goodenough, D.A. 1993. Multisubunit assembly of an integral plasma membrane channel protein, gap junction connexin 43 , occurs after exit from the ER. Cell. 74:1065-1077.

22. Greger, R. 1985. Ion transport mechanisms in thick ascending limb of Henle's loop of mammalian nephron. Physiol. Rev. 65:760-797.

23. Kiuchi-Saishin, Y., et al. 2002. Differential expression patterns of claudins, tight junction membrane proteins, in mouse nephron segments. J. Am. Soc. Nephrol. 13:875-886.

24. Van Itallie, C.M., et al. 2006. Two splice variants of claudin-10 in the kidney create paracellular pores with different ion selectivities. Am. J. Physiol. Renal Physiol. 291:F1288-F1299.

25. Fujimoto, K. 1995. Freeze-fracture replica electron microscopy combined with SDS digestion for cytochemical labeling of integral membrane proteins. Application to the immunogold labeling of intercellular junctional complexes. J. Cell Sci. 108:3443-3449 